\title{
Un 25 aprile a distanza: la Resistenza nella scuola primaria attraverso la DAD
}

\author{
di Chiara Dogliotti
}

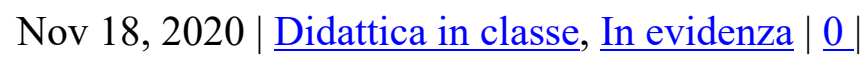

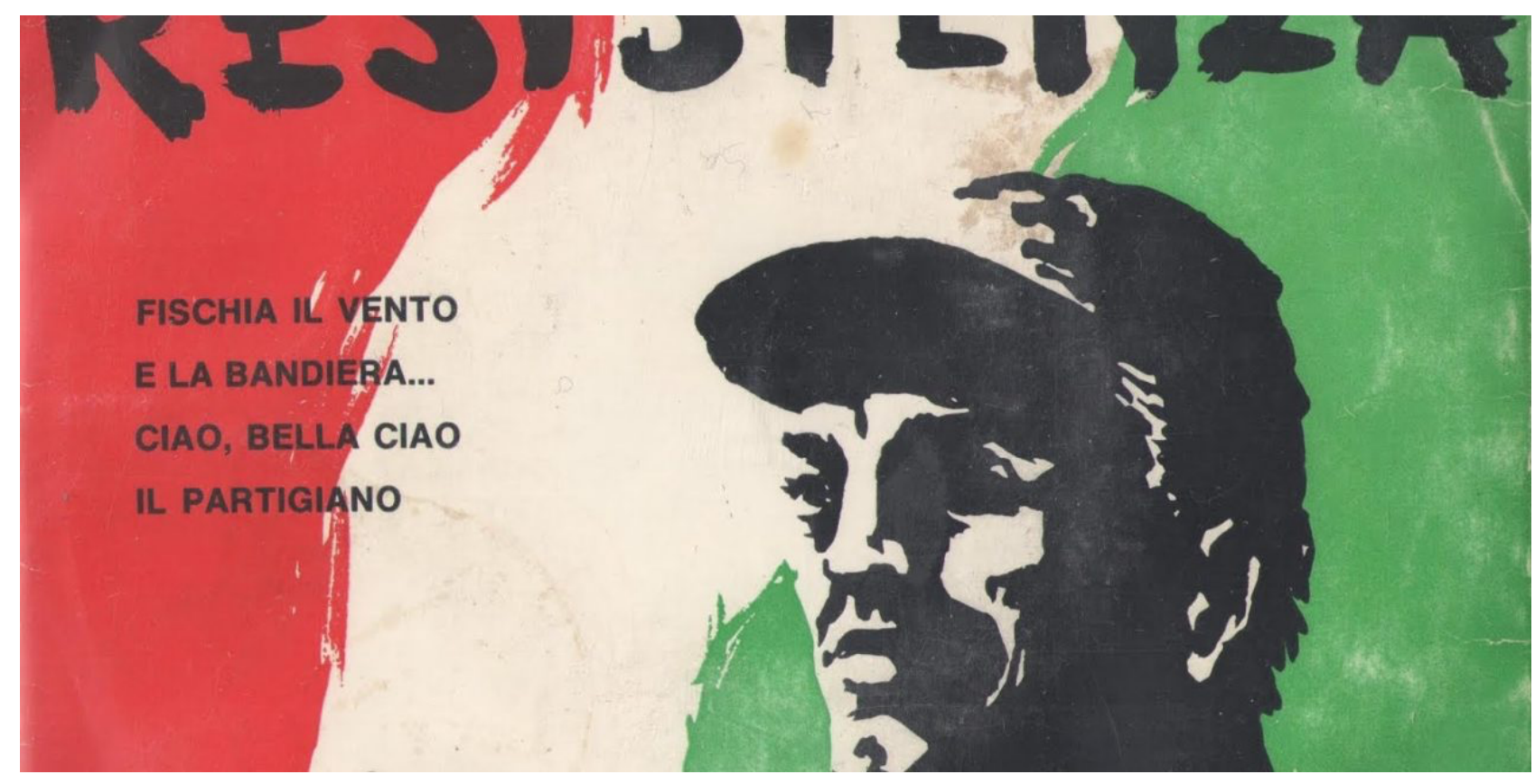

Copertina di un disco con canti partigiani inciso negli anni '60

\begin{abstract}
L'articolo propone l'esperienza didattica effettuata nelle classi quarta e quinta di scuola primaria in provincia di Genova con la Didattica a Distanza nella primavera del 2020. Si tratta di un progetto che attraverso l'analisi dei canti partigiani e di una biografia sulla scorta di immagini e documenti veniva precedentemente svolto con modalità tradizionale e che è stato adattato alla situazione di chiusura totale per permettere una riflessione sul 25 aprile.
\end{abstract}

La sfida della didattica a distanza

La didattica a distanza (DAD) è una sfida particolarmente complessa nella scuola primaria per le molte criticità legate alla giovanissima età dei discenti: scarsa autonomia nell'esecuzione e nello studio, limitata alfabetizzazione tecnologica e digitale, rischi legati alla lunga permanenza dei bambini davanti al computer. Tuttavia, la pratica della didattica a distanza ha costituito, laddove sia stata messa in atto realmente e con cognizione delle sue potenzialità didattiche, il mezzo per mantenere le relazioni nel gruppo classe e il legame con la scuola; inoltre ha rivelato anche aspetti positivi didattici e relazionali che sarebbe importante conservare anche nella fondamentale didattica in presenza.

Per quanto riguarda i bambini della primaria, è sicuramente vero che le loro skill informatiche non sono generalmente molto avanzate e che la permanenza davanti al computer può risultare stressante; tuttavia queste criticità sono almeno in parte compensate dai benefici psicologici e didattici 
derivanti dal mantenere vivo il legame con la vita scolastica e la continuità del percorso formativo. Bisogna anche evitare di sottostimare la competenza digitale dei bambini; in particolare, quella di bambini provenienti da contesti culturali poco stimolanti in cui è diffusa l'abitudine di trascorrere molto tempo davanti a tablet e smartphone sia per giocare, sia per utilizzare i social e quelle di figli di genitori immigrati che utilizzano frequentemente la tecnologia per comunicare con nonni, amici e parenti lontani. Non stupisce quindi constatare che a trarre particolare beneficio dal lavoro in classe virtuale siano stati nella nostra esperienza molti bambini che nelle aule reali mostravano importanti difficoltà derivanti o da barriere linguistiche vere e proprie o da quelle determinate da quel gap culturale che aveva ben evidenziato già don Milani quando notava che per i figli dei contadini quella della scuola è una lingua straniera. Nell'aula virtuale le abilità tecnologiche di questi alunni si sommano ad altri elementi (l'assenza di fattori di distrazione e disturbo, la possibilità di interrompere il flusso di parole di un video o di una registrazione vocale, il tempo più dilatato di decodifica, l'opportunità di prepararsi in anticipo rispetto alla "lezione" in videochat con i materiali forniti attraverso la piattaforma digitale) creando un ambiente di apprendimento adeguato ai loro bisogni.

\section{Didattica a distanza e diseguaglianze}

Il lavoro da remoto ha messo in evidenza, e si tratta certamente di un aspetto su cui riflettere, le diseguaglianze sociali.

Il problema, infatti, preesisteva e la sua emersione prepotente è legata non solo e non tanto al numero di giga o all'efficienza dei computer di cui ogni bambino dispone, quanto alla riduzione del tempo trascorso a scuola a favore di quello trascorso in famiglia. La necessità di raggiungere tutti i bambini ha, però, fatto sì che i docenti dovessero trovare nuove e più efficaci strategie per superare i gap culturali, sociali ed economici delle famiglie in situazioni difficili. Nella nostra esperienza, i ragazzi, chiamati in videochat a condividere con i compagni il risultato di lavori assegnati dalla scuola e pensati per essere alla portata di tutti, perché tutti avessero il proprio obiettivo da raggiungere, apparivano soddisfatti del proprio impegno e dei risultati raggiunti, a proprio agio con sé stessi e con il gruppo. Inoltre, attraverso l'organizzazione di un piano di lavoro a distanza, con scadenze e appuntamenti regolari, si è andati incontro a quel bisogno di routine che è tipico di tutti $i$ bambini, sollevando i genitori da un compito oneroso e non di loro competenza come è quello della didattica e al contempo assicurando agli alunni una rassicurante continuità con la vita scolastica. Questo valore della DAD è ancora più importante per gli studenti portatori di disabilità, in particolare per i casi di autismo in cui la predisposizione di rituali e il mantenimento di abitudini e relazioni consolidate sono indispensabili per l'equilibrio e il benessere psicofisico.

\section{Entrare nelle case}

Infine, il fatto di entrare nelle case attraverso la DAD ha rafforzato l'alleanza educativa tra docenti e famiglie: si è collaborato più strettamente e le famiglie hanno avuto modo di conoscere e apprezzare maggiormente il lavoro degli insegnanti; in questo modo sia la fiducia reciproca, sia il rapporto ne sono usciti consolidati. Da questo punto di vista la didattica a distanza ha offerto agli insegnanti una grande occasione di riflessione sulla propria capacità di relazionarsi con le famiglie e con i loro bisogni e di reimpostare, laddove fosse necessario, questa relazione, imparando a considerare le famiglie come risorse, rinforzando la collaborazione tra scuole e case nell'interesse dei ragazzi. 


\section{Un 25 aprile a distanza}

Come esempio delle possibilità offerte dalla DAD nella scuola primaria proponiamo un percorso didattico rivolto alle classi quarte e quinte e realizzato in una scuola primaria del centro storico di Genova intitolato "Un bambino nella resistenza": attraverso la riproduzione fotografica di documenti originali (lettere, fotografie e diari), il racconto di testimonianze e l'impiego di materiali multimediali (canzoni, video, mappe geografiche satellitari) si intraprende un viaggio da una storia alla storia. [le immagini utilizzate sono raccolte nel Power Point che si allega].

Per poter dare vita a un progetto didattico a distanza sulla Resistenza si è dovuto a ricorrere a numerosi diversi strumenti che la tecnologia ci mette a disposizione a partire dalle video chat attraverso cui abbiamo cominciato a introdurre l'argomento all'inizio di aprile, conducendo brevi brain storming sviluppati intorno a questioni quali: "Sapete perché il 25 aprile è festa?", "I vostri genitori o i vostri nonni vi hanno mai parlato della Resistenza?", "Avete mai visto qualche film o qualche documentario sulla Resistenza o sulla Seconda guerra mondiale?", "Conosci la canzone Bella ciao? Sai di cosa parla?"

Da queste discussioni sono emerse principalmente tre cose: 1'argomento non era completamente estraneo ai bambini, molti dei quali avevano ascoltato racconti o letto storie o guardato documentari e fiction; nonostante questa relativa familiarità col tema la confusione era grande sotto il cielo e le informazioni risultavano piuttosto confuse e poco contestualizzate, quasi tutti conoscevano la canzone Bella ciao e molti avevano apprezzato particolarmente storie raccontate dal punto di vista di bambini.

\section{Canzoni partigiane}

Con i colleghi di team Daniele Andreallo, Mara Dedola e Giulia Tarabotto, si è quindi deciso di iniziare dalle canzoni partigiane, elaborando un percorso che partisse proprio dalla più nota Bella ciao. Nella prima videolezione abbiamo ascoltato assieme alcune versioni di questo canto per poi aprire sulla piattaforma della classe virtuale una sezione dedicata al 25 aprile in cui è stata inserita una cartella denominata 10,100,1000 Bella ciao in cui si sono caricati link a diverse interpretazioni del brano con particolare attenzione alle versioni in lingue diverse. Nella videolezione successiva è stata presentata una diversa canzone - Dalle belle città - raccontando che fu composta nel marzo del 1944 dai partigiani Emilio "Cini" Casalini e Angelo "Lanfranco" Rossi, appartenenti al 5 distaccamento della III Brigata Garibaldi "Liguria" in un territorio molto vicino alla città degli alunni: sull'Appennino ligure-piemontese, nella zona del Monte Tobbio. A questo punto ho messo in condivisione la pagina di Google con cui abbiamo cercato la voce "Monte Tobbio" per visualizzarla attraverso Google map, analizzare le caratteristiche del territorio e calcolare la distanza tra quella zona e la nostra scuola. Questa attività ha permesso di introdurre una serie di considerazioni sul contesto della lotta partigiana non semplicemente tramesse, ma costruite insieme alla classe attraverso un ragionamento collettivo: quali furono i luoghi in cui era più diffusa la presenza dei partigiani e perché, quali caratteristiche ha la guerra asimmetrica contro un esercito di occupazione, quali rischi correvano i partigiani, l'importanza del controllo di vie di comunicazione e confini e così via. Come completamento e approfondimento di questa prima parte del progetto è stato caricato sulla sezione 25 aprile della nostra classe virtuale il link a una pagina del sito dell'Isral in cui Franco Castelli propone la genesi e l'analisi del canto[1] e a diverse versioni di gruppi musicali contemporanei. 


\section{Un bambino nella resistenza}

La seconda parte del progetto è costruita intorno a un racconto; si tratta di una storia vera, ambientata tra Genova e la vicina Val Trebbia, che ha per protagonista un bambino pressappoco coetaneo degli alunni della classe. [vedi Power Point che si allega].

La storia del bambino e dei personaggi a lui collegati - il padre membro del CLN, la madre antifascista, il fratello partigiano, una sorella prigioniera delle SS, un'altra sorella infermiera partigiana - fornisce numerosi spunti per offrire elementi di contesto generale del fenomeno; il racconto si è svolto nel corso di incontri in videochat dedicati che avvenivano una volta a settimana e con il supporto della riproduzione fotografica di documenti provenienti dall'archivio privato della famiglia protagonista della narrazione: fotografie, lettere, pagine di diari, documenti militari, onorificenze e così via.

Al termine del racconto si è divisa la classe in coppie ad ognuna delle quali è stato assegnato uno dei documenti riprodotti e inviato tramite allegato di messaggio di Moodle[2] con la consegna di collaborare nella scrittura di un episodio o del ritratto di un personaggio della storia; la comunicazione tra i ragazzi poteva avvenire tramite telefonate o invio di mail o videochat e gli scritti andavano restituiti attraverso la funzione "compito" della classe virtuale. Gli scritti consegnati sono stati quindi rielaborati graficamente e abbinati alle immagini costruendo un power point intitolato "Un bambino nella Resistenza". La partecipazione alla stesura di questa sorta di libro collettivo della classe, il cui risultato finale è stato presentato in una videochat conclusiva ed è stato quindi caricato nella cartella della classe virtuale dedicata al progetto, ha molto gratificato i ragazzi che hanno dimostrato soddisfazione per la restituzione del proprio prodotto e hanno gradito la possibilità di lavorare in coppia con un compagno seppure a distanza, sperimentando di nuovo così almeno in parte la relazione coi pari. A titolo esemplificativo, alcuni dei prodotti realizzati.

[inserire qui i lavori dei ragazzi]

\section{Bilancio e prospettive}

Il percorso continuerà il prossimo anno con un incontro - in presenza o a distanza - con il protagonista del racconto ormai ottantenne che porterà ai bambini la sua testimonianza, in modo che la storia emersa dalle fonti documentarie trovi una voce narrante viva attraverso la fonte orale.

Se la valutazione del lavoro svolto a casa in autonomia, per quanto in collaborazione con un compagno, risulta difficoltosa in quanto è arduo stabilire quanto abbiano inciso un ipotetico intervento degli adulti e la possibilità o meno di consultare libri o siti web, è stato invece agevole avere il polso della situazione della ricezione del progetto attraverso gli incontri in videochat e $i$ dibattiti che si sono svolti in quelle occasioni. Il bilancio ricavabile da queste verifiche è molto positivo: gli interventi sono stati sempre numerosi e pertinenti; le domande molto frequenti e rivelatrici di attenzione, comprensione e interesse per il tema; commenti, silenzi e risate durante il racconto dimostravano un ascolto attivo; infine, il controllo degli accessi ai contenuti della cartella 25 aprile ha mostrato che circa i due terzi della classe hanno dedicato tempo all'approfondimento dell'argomento proposto. 
Le canzoni partigiane e il racconto di aneddoti avventurosi o divertenti inquadrati in una storia di cui è protagonista un bambino avevano lo scopo di suscitare l'interesse dei ragazzi e di comunicare loro i valori civili e lo spirito democratico della Resistenza; nello stesso tempo, queste parole, immagini e musica offrivano gli spunti adeguati per introdurre alcuni semplici, ma fondamentali elementi di contesto storico della vicenda: spazi e tempi, innanzitutto, poi il rapporto tra partigiani e popolazione e quello tra resistenza armata e civile, lo sfondo della seconda guerra mondiale, dell'occupazione tedesca e il ruolo del fascismo repubblicano. Piuttosto inaspettatamente, durante le videochat, il discorso si è approfondito fino a toccare questioni più complesse e profonde come le ragioni della scelta delle armi e la falsità del mito del "buon italiano".

Questo percorso didattico è frutto di un adattamento in tempi di didattica a distanza di un percorso svolto in classe; i limiti, rispetto al progetto originale, sono soprattutto legati ai tempi di svolgimento, necessariamente più stretti per evitare lunghe permanenze dei bambini davanti al monitor, che hanno quindi comportato una compressione dei momenti di lezione e di laboratorio nel grande gruppo. Nonostante questa criticità, è stato possibile raggiungere tutti gli obiettivi didattici, etici e relazionali che il percorso si prefiggeva, grazie alla combinazione di diversi strumenti offerti dalla tecnologia (piattaforma per la classe digitale, videochat, programmi di videoscrittura e per la creazione di power point, mappe digitali, siti internet) e alla competenza digitale degli alunni che hanno consentito di non interrompere il filo della vita scolastica, di portare avanti i progetti didattici programmati e di non lasciare indietro alcun ragazzo.

\section{Note:}

[1] https://www.isral.it/risorse-e-documenti/25-aprile-la-liberazione-e-il-movimentopartigiano/materiali-documenti-e-testimonianze/un-canto-nato-alla-benedicta-siamo-i-ribelli-dellamontagna/.

[2] Moodle (http://www.moodle.org ) è una Piattaforma open source molto utilizzata per la formazione a Distanza, cioè un pacchetto software per erogare e gestire corsi di formazione on-line. 